\title{
Glycan Shedding in both the Blood and CSF: Initial Predictor of Immune Attack in Autoimmune Encephalomyelitis?
}

\section{Shanshan Pei}

Southern Medical University Nanfang Hospital

Jiajia Zhu

Southern Medical University Nanfang Hospital

\section{Zheyi Zhou}

Liuzhou Traditional Chinese Medical Hospital

\section{Yuewen Ding}

Southern Medical University Nanfang Hospital

Yu Peng

Southern Medical University Nanfang Hospital

Zhanhang Wang

Southern Medical University Nanfang Hospital

\section{Jinyu Chen}

Southern Medical University Nanfang Hospital

honghao wang ( $\nabla$ wang_whh@163.com )

Southern Medical University Nanfang Hospital https://orcid.org/0000-0002-1215-6219

\section{Research}

Keywords: glycocalyx; blood-brain barrier; cerebrospinal fluid; neuromyelitis optica; disease severity

Posted Date: January 29th, 2020

DOI: https://doi.org/10.21203/rs.2.22182/v1

License: (9) (i) This work is licensed under a Creative Commons Attribution 4.0 International License. Read Full License 


\section{Abstract}

Background Neuromyelitis optica (NMO), multiple sclerosis (MS) and autoimmune glial fibrillary acidic protein (GFAP) astrocytopathy belong to autoimmune diseases incentral nervous system mainly manifestate encephalomyelitis. The glycocalyx (GLX), consists of several membrane-bound macromolecules, is located on the luminal side of the endothelium and mediates the blood and the vessel interaction. Until now, there is still lacking a holistic understanding of the GLX degradation in autoimmune encephalomyelitis. Aim This study aimed to detect the shedding levels of GLX components, heparan sulfate (HS) and hyaluronic acid (HA) in serum and cerebrospinal fluid (CSF), correlate them with the severity and assess the diagnostic value of them, and evaluate their correlations with pro-inflammatory cytokines. Methods Serum and CSF samples were obtained from 24 NMO patients, $15 \mathrm{MS}$ patients, 10 autoimmune GFAP astrocytopathy patients, and 18 controls without non-inflammatory neurological diseases. Soluble HS, HA and IFN- $y$, IL-17A, matrix metalloproteinase (MMP)-1 were detected by enzyme linked immunosorbent assay ELISA. Results Besides levels of serum and CSF levels of HS, HA and related cytokines were significantly elevated in these diseases. Notably, HS, HA in NMO, MS patients, and autoimmune GFAP astrocytopathy diseases were widely correlated with EDSS scores. Importantly, the ROC curve analysis suggested a potential diagnostic role of HS or HA . Conclusions The results here suggested the GLX degradation and inflammation in NMO, MS and autoimmune GFAP astrocytopathy. Moreover, increased shedding of HS or HA may indicate worse clinical situation. Importantly, CSF HS and HA may be informative diagnostic biomarkers for telling autoimmune encephalomyelitis from the non-inflammatory neurological controls. Furthermore, therapeutic strategy for protecting GLX may be effective to these diseases.

\section{Introduction}

Neuromyelitis optica (NMO), multiple sclerosis (MS) and autoimmune glial fibrillary acidic protein (GFAP) astrocytopathy belong to autoimmune diseases incentral nervous system [1]. NMO and MS are two representative immune-mediated AE [2]. Autoimmune GFAP astrocytopathy is a newly with unclear pathogenesis and no accurate diagnostic criteria [3]. Typically, the denominator of these antibody-mediated diseases involves lymphocyte infiltration, astrocytopathy and autoantibodies targeting the receptors in the brain or spinal cords $[4,5]$. Considering the integrity of blood-brain barrier (BBB) prevents the entry of immune cells and antibodies into the CNS [6]. Moreover, diverse neuro-inflammation is the basic pathologic change of NMO, MS and autoimmune GFAP astrocytopathy closely linked to the breakdown of BBB [7], the interplay of neuro-inflammation and BBB dysfunction may seriously result in neurological disturbance $[8,9]$. To date, the state of BBB in these diseases is not fully demonstrated, and the exploration of this issue may facilitate the understanding of these diseases and offer novel therapies.

Glycocalyx (GLX), a layer consisted of polysaccharides and proteoglycans and attached to the luminal side of endothelium of blood-brain barrier (BBB), acts as the first barrier between blood flow and the BBB [10]. Under the physiological conditions, GLX is in a state of dynamic equilibrium that is continuously degraded and continuously synthesized $[11,12]$. Because of the vulnerability to various pathological conditions like inflammation, hypertension and edema, components of GLX will be sensitively and quickly fragmented and released into CSF or bloodstream [13-15]. Once the dynamic equilibrium is broken, diverse diseases such as vascular, renal, and inflammatory diseases may be aggravated $[13,16]$. In response to pathological conditions, extracellular proteases quickly get activated and mediates the shedding of components of GLX, resulting in vascular permeability barrier break, mechanotransduction impairment and endothelial cell dysfunction, and these changes conversely enhanced 
the degradation of glycocalyx in a vicious circle [17]. Till now, most researches concerning GLX focus on vascular diseases and cancer. Though a few papers have connected the components of GLX with encephalitis like NMO, EAE and anti-NMDAR encephalitis [18-20], there is still lacking a holistic understanding of the GLX degradation in autoimmune encephalomyelitis.

Glycocalyx is a delicate membrane-bound network whose components includes glycosaminoglycans (GAGs) and proteoglycans (PGs). HS and HA are two representative molecules belongs to GAGs and their degradation indicating endothelial glycocalyx degradation [10, 21, 22]. In the early stage of immune attack, pro-inflammatory cytokines can be immediately derived from $\mathrm{T}$ cells and their activation quickly stimulate the generation and maturation of MMPs, a kind of known protease for GLX [23-25]. Indeed, previous studies concerning other diseases like sepsis, brain edema, stroke, and trauma have confirmed the GLX shedding in diseases' acute stage $[17,26,27]$. Thus, plasma and CSF concentrations of HS and HA can largely reflex the damage of GLX, and indirectly the disruption of BBB in acute stage. Until now, no studies have published the detailed information on shedding of the glycosaminoglycans (GAGs), particularly HS and HA, in CSF and serum in patients with encephalomyelitis of NMO, MS and autoimmune GFAP astrocytopathy.

Herein, we focus on the role of glycocalyx in encephalomyelitis of NMO, MS and autoimmune GFAP astrocytopathy, three typical antibody-dependent astrocytic disorders. To assess the influence of the severity of BBB injury on the severity of these neurological disorders, and find out the potential factors affecting the integrity of BBB glycocalyx, we detected the levels of two molecules of glycocalyx, HS and HA, in both CSF and serum, correlated them with CSF levels of pro-inflammatory factors and sheddases, IFN- $\gamma$, IL-17A, MMP-1, or EDSS scores. Our results showed a dramatic increase of CSF and plasma concentrations of HS and HA, and elevated levels of CSF IFN- $\gamma$, IL-17A, MMP-1 in these three autoimmune encephalomyelitis. More importantly, the shedding levels of glycocalyx molecules in CSF positively affected the severity of NMO, MS and autoimmune GFAP astrocytopathy, and the degree of inflammation may aggravate the disruption of the blood-brain barrier. However, the dynamic concentrations of HS and HA and their relationship with severity in different course of these disorders remain unknown. Of note, CSF HS and HA may be reliable markers for the diagnosis of encephalomyelitis of NMO, MS and autoimmune GFAP astrocytopathy. Further more, therapeutic strategies focused on preservation of glycocalyx may improve outcomes of these neurological disorders.

\section{Methods And Materials}

\subsection{Patients and clinical assessments}

All subjects were enrolled from the Department of Neurology of Nanfang Hospital, Southern Medical University, including 24 NMO patients, 15 MS patients, 10 patients with autoimmune GFAP astrocytopathy and 18 controls without inflammatory or autoimmune neurological diseases (peripheral neuropathy $=10$, movement disorder $=6$, Alzheimer's disease $=2$ ). The diagnoses of the above autoimmune encephalitis were confirmed blindly by two doctors based on the diagnostic criteria $[3,28,29]$. All controls were negative for specific CSF and serum antibodies. EDSS score was used to evaluate the severity of patients. In addition, relevant demographic and medical data were also collected, shown in Table 1. By the way, this study was approved by the Ethics Committee of the Nan Fang Hospital and all the above subjects have signed the informed consent. 
Table 1

Demographic and clinical features of the patients and controls.

\begin{tabular}{|c|c|c|c|c|}
\hline & CLTs $(n=18)$ & NMO ( $n=24)$ & MS (n=15) & $\begin{array}{l}\text { autoimmune GFAP } \\
\text { astrocytopathy }(\mathrm{n}= \\
\text { 10) }\end{array}$ \\
\hline $\begin{array}{l}\text { Gender } \\
\text { (female/male) }\end{array}$ & $11 / 7$ & $16 / 8$ & $11 / 4$ & $6 / 4$ \\
\hline Age (years)a & $35.43 \pm 10.32$ & $34.37 \pm 15.24$ & $36.27 \pm 14.75$ & $33.46 \pm 17.27$ \\
\hline EDSS scoresb & - & $4.0(3.13,4.88)$ & $2.5(2.00,3.00)$ & $2.75(2.38,3.50)$ \\
\hline $\begin{array}{l}\text { Serum HS } \\
(\mathrm{ng} / \mathrm{mL}) \mathrm{b}\end{array}$ & 236.8(164.2, 320.5) & $\begin{array}{l}429.4(292.7 \\
523.8)^{\star \star \star}\end{array}$ & $\begin{array}{l}321.6(254.6 \\
390.0)^{\star}\end{array}$ & $395.8(316.3,508.4)^{\star \star \star}$ \\
\hline $\begin{array}{l}\text { CSF HS } \\
(\mathrm{ng} / \mathrm{mL}) \mathrm{b}\end{array}$ & $96.9(74.9,135.2)$ & $\begin{array}{l}219.4(148.2 \\
272.5)^{\star \star \star}\end{array}$ & $\begin{array}{l}\text { 183.7(133.5 } \\
202.4)^{\star \star \star}\end{array}$ & $207.6(158.2,259.7)^{\star \star \star}$ \\
\hline $\begin{array}{l}\text { Serum HA } \\
(\mathrm{ng} / \mathrm{mL}) \mathrm{b}\end{array}$ & $26.5(24.4,37.2)$ & $70.4(50.0,88.4)^{\star \star \star}$ & $66.8(51.10,85.9)^{\star \star \star}$ & $71.9(48.6,76.9)^{\star \star \star}$ \\
\hline $\begin{array}{l}\text { CSF HA } \\
(\mathrm{ng} / \mathrm{mL}) \mathrm{b}\end{array}$ & $32.5(26.9,36.0)$ & $\begin{array}{l}48.1(40.75 \\
57.02)^{\star \star \star}\end{array}$ & $\begin{array}{l}49.04(39.71 \\
56.14)^{\star \star \star}\end{array}$ & $47.5(28.6,61.6)$ \\
\hline $\begin{array}{l}\text { Serum IFN-y } \\
(\mathrm{pg} / \mathrm{mL}) \mathrm{b}\end{array}$ & $8.2(5.7,14.2)$ & 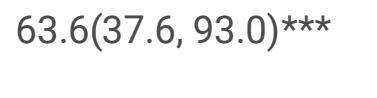 & $54.4(34.9,94.8)^{\star \star \star}$ & $57.2(41.6,84.0)^{\star \star \star}$ \\
\hline $\begin{array}{l}\text { CSF IFN-y } \\
(\mathrm{pg} / \mathrm{mL}) \mathrm{b}\end{array}$ & $8.7(5.4,12.6)$ & $73.0(43.5,86.4)^{\star \star \star}$ & $44.8(24.5,80.6)^{\star \star \star}$ & $38.8(29.4,58.6)^{\star \star \star}$ \\
\hline $\begin{array}{l}\text { Serum IL-17A } \\
(\mathrm{pg} / \mathrm{mL}) \mathrm{b}\end{array}$ & $2.7(2.55,3.2)$ & $5.5(4.5,7.7)^{\star \star \star}$ & $5.3(3.7,9.2)^{\star \star \star}$ & $7.0(4.5,7.6)^{\star \star \star}$ \\
\hline $\begin{array}{l}\text { CSF IL-17A } \\
(\mathrm{pg} / \mathrm{mL}) \mathrm{b}\end{array}$ & $3.2(3.1,3.4)$ & 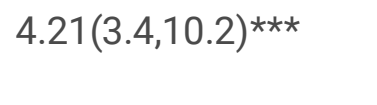 & $5 \cdot 2(4 \cdot 6,8.6)^{\star \star \star}$ & $6.1(3.7,10.3)^{\star \star \star}$ \\
\hline $\begin{array}{l}\text { Serum MMP-1 } \\
(\mathrm{pg} / \mathrm{mL}) \mathrm{b}\end{array}$ & $598.8(236.3,1050.0)$ & $548.3(341.5,1512.0)$ & $992.7(491.2,2837.0)$ & $631.1(354.3,976.9)$ \\
\hline $\begin{array}{l}\text { CSF MMP-1 } \\
(\mathrm{pg} / \mathrm{mL}) \mathrm{b}\end{array}$ & $14.8(13.3,15.7)$ & $17.8(15.3,23.7)^{\star \star}$ & $\begin{array}{l}19.28(16.49 \\
20.4)^{\star \star \star}\end{array}$ & $22.6(18.3,30.8)^{\star \star \star}$ \\
\hline \multicolumn{5}{|l|}{ NMO-IgG } \\
\hline Positive & 0 & 23 & 0 & 0 \\
\hline \multicolumn{5}{|l|}{ GFAP-IgG } \\
\hline Positive & 0 & 0 & 0 & 10 \\
\hline \multicolumn{5}{|c|}{$\begin{array}{l}\text { Age (years) refers to age at sample collection. } n \text { = number; IL, interleukin; TNF, tumor necrosis factor; CSF, } \\
\text { Cerebrospinal Fluid; EDSS, Expanded Disability Status Scale; NA: not available; HS: heparan sulphate; HA: } \\
\text { hyaluronic acid; NMO, neuromyelitis optica; MS, multiple sclerosis. GFAP: glial fibrillary acidic protein. }\end{array}$} \\
\hline \multicolumn{5}{|c|}{ a. Data were presented as mean \pm SD. SD: standard deviations. } \\
\hline \multicolumn{5}{|c|}{ b. Data were presented as medians (IQRs-interquartile ranges). } \\
\hline$\star P<0.05, * \star P<$ & $1, * \star \star P<0.001$ & & & \\
\hline
\end{tabular}


We obtained both the CSF and serum samples from all subjects within the 3 days after admission and before the Immunotherapy therapies were applied. Then all samples went through a mild centrifuge and stored at $-80^{\circ} \mathrm{C}$ until assay. Concentrations of HS, HA and related cytokines like IFN- $\gamma$, IL-17A and MMP-1 were detected by Enzyme-Linked Immunosorbent Assay (ELISA) kits (R\&D Systems). All detections were performed in accordance with the manufacturer's instructions and every standard and sample were assayed in duplicate.

All statistical analyses were conducted using SPSS version 24.0 (IBM, Armonk, NY, US). Data were displayed as the mean \pm SEM or median (IQRs-interquartile ranges) according to the normality test results. Kruskal-Wallis plus Dunn's test or one-way analysis of variance (ANOVA) with Tukey post hoc analysis were performed accordingly. $p<$ 0.05 was taken as statistically significant. Graphs were plotted by GraphPad Prism 8 (GraphPad, La Jolla, CA, US). All analyses were performed in a blinded manner.

\section{Results}

\subsection{Demographic and Clinical characteristics}

The data derived from patients with NMO $(n=24), M S(n=15)$, GFAP $(n=10)$ and 18 controls were presented in Table 1. The median (IQRs) EDSS score was $4.0(3.13,4.88)$ for the NMO group, $2.5(2.00,3.00)$ for the MS group and $2.75(2.38,3.50)$ for the GFAP group (Table 1$)$. No statistically significant differences on sex or age in each group.

3.2 Increased HS, HA and related cytokine levels in serum and CSF in patients with autoimmune encephalomyelitis

As shown in Table 1, the median plasma concentrations of IFN- $\gamma$ and IL-17A were all obviously elevated in patients with NMO and MS in comparison with that of the control subgroup (IFN- $-\mathrm{p}<0.001, p<0.001$, respectively; IL-17A: $p<0.001, p<0.001$, respectively; MMP-1: $p<0.001, p<0.001$, respectively). For autoimmune GFAP astrocytopathy, plasma concentrations of IFN- $\gamma$ and IL-17A but not MMP-1 were higher than that of controls (IFN-y: $p<0.001$; IL17A: $p<0.001)$. Similarly, the median CSF concentrations of IFN- $\gamma$, IL-17A and MMP-1 were all obviously elevated in patients with NMO, MS and GFAP in comparison with that of the control subgroup (IFN-y: $p<0.001, p<0.001, p<$ 0.001, respectively; IL-17A: $p<0.001, p<0.001, p<0.001$, respectively; $p$; MMP-1: $p<0.001, p<0.001, p<0.001$, respectively). To further assess the BBB permeability injury severity in these autoimmune encephalitis patients, we compared the levels of HS and HA in patients with NMO, MS and autoimmune GFAP astrocytopathy as well as controls. The results shown in Fig. 1 demostrated that concentrations of both HS and HA in plasma and CSF were elevated in the autoimmune encephalitis group compared with the control group (Serum HS: $p<0.001, p=0.022, p$ $<0.001$, respectively; CSF HS: $p<0.001, p<0.001, p<0.001$, respectively; Serum HA: $p<0.001, p<0.001, p<0.001$, respectively; CSF HA: $p=0.057, p<0.001, p<0.001$, respectively). However, no significant differences were found in levels of serum or CSF glycocalyx molecules and other cytokine parameters among NMO, MS and autoimmune GFAP astrocytopathy patients. Further, to find out the relationship between levels of CSF and plasma glycocalyx molecules, correlations were tested in autoimmune encephalitis subgroups, of which only HS in NMO and HA in MS appeared a positive correlation between CSF and serum levels (NMO HS: $p=0.029, r=0.447$; MS HA: 0.005, $0.696)$ (S 2).

3.3 Correlations between levels of CSF and plasma HS and HA and EDSS scores 
To assess the possible link between CSF and plasma glycocalyx molecules and the severity of these three disorders, we then examined the correlations (Fig. 2). Of note, in patients with NMO, MS or autoimmune GFAP astrocytopathy, we discovered a significant positive correlation between concentrations of CSF HS, HA and EDSS (NMO HS: $p=0.024, r=460$; MS HS: $p=0.004, r=707$; GFAP HS: $p=0.047, r=650$; NMO HA: $p=0.010, r=514$; MS HA: $p=0.030, r=568 ;$ GFAP HA: $p=0.037, r=675)$. As for associations between plasma profiles levels and EDSS scores, we only found a significant correlation between serum HS and EDSS in NMO subgroup $(p=0.043, r=$ 0.417). Results showed positive correlations between other serum levels and EDSS, though they did not quite reach statistical significance (NMO HA: $p=0.051, r=403$; MS HS: $p=0.064, r=495$; GFAP HS: $p=0.241, r=409 ;$ MS HA: $p=0.104, r=440 ;$ GFAP HA: $p=0.065, r=613)$.

\subsection{Associations between CSF glycocalyx molecules and other cytokine parameters}

Previous studies have found elevated levels of pro-inflammatory cytokines in CSF of patients with NMO, MS and autoimmune GFAP astrocytopathy. Here, we further detected the associations between CSF glycocalyx molecules and IFN- - , IL-17A and MMP-1 (Fig. 3). Interestingly, in the CSF of patients with NMO, MS and autoimmune GFAP astrocytopathy, there was a universal positive correlation between concentrations of HS and that of MMP-1 (NMO, $p=0.032, r=0.483$; MS, $p=0.024, r=0.586$; GFAP, $p=0.025, r=0.711$ ) (Fig. 3A, C, E). Moreover, significant positive correlations were also found between CSF HS and IFN- $\gamma(p=0.022, r=0.465)$ and CSF HA and MMP-1 ( $p=0.001, r$ $=0.622)$ in NMO subgroup (Fig. 3A, B), CSF HS and IL-17A $(p=0.043, r=0.533)$ and CSF HA and IFN- $y(p=0.014, r$ $=0.629)$ in MS subgroup (Fig. $3 C, D)$, and CSF HA and IL-17A ( $p=0.035, r=0.685)$ in autoimmune GFAP astrocytopathy subgroup (Fig. 3F).

\subsection{Diagnostic values of soluble HS and HA for the assessment of disease activity}

To measure the diagnostic value in distinguish controls and NMO, MS and autoimmune GFAP astrocytopathy, we conducted ROC curve analysis. As shown in Fig. 4, both CSF HS and HA showed good area under the curve (AUC) values (NMO HS, 0.891; MS HS, 0.880; GFAP, 0.919; NMO HA, 0.852; MS HS, 0.885; GFAP, 0722), among which the AUC of CSF HS in autoimmune GFAP astrocytopathy and CSF HA in MS were the supreme, whose optimal cut-off values were 155.9 and $37.72 \mathrm{ng} / \mathrm{mL}$, indicating the highest diagnostic potential of CSF HS for autoimmune GFAP astrocytopathy and CSF HA for MS. In the comparison of patients with NMO, MS and GFAP encephalitis, the AUC were $0.658,0.504,0.660$ for CSF HS and $0.502,0.563,0.573$ for CSF HA, respectively. Of note, HS and HA in CSF did not show an improved diagnostic value for distinguishing NMO, MS and GFAP (S 1).

\section{Discussion}

This study focused on the disruption extent of glycocalyx (GLX) in three typical autoimmune encephalomyelitis, NMO, MS and autoimmune GFAP astrocytopathy, which was reflexed by the shedding levels of HS and HA in plasma and CSF here. Further, we found the diagnostic role of HS and HA in these three autoimmune diseases in CNS.

As is known to us all, the BBB prevents certain substances like inflammatory factors and immune cells in plasma from entering the central nervous system $[13,15,30]$, thus BBB plays a crucial role in keeping cerebral homeostasis. Moreover, evidence have suggested the breakdown of BBB in autoimmune diseases of CNS [31], but the early changes of BBB milieu still largely remain unclear in encephalomyelitis, in particular the newly identified 
autoimmune GFAP astrocytopathy. Importantly, the effective therapeutic protecting the BBB in other diseases like brain edema indicated the potential therapy for NMO, MS and autoimmune GFAP astrocytopathy [17].

GLX, a jelly-like layer, encompasses the luminal surface of the endothelium and has been taken as the first defense of BBB $[10,17,32]$. Once this surface matrix is disrupted, the homeostasis of endothelial permeability will be broken, resulting in leakage of inflammatory cytokines, immune cells and even antibodies from serum to cerebrospinal fluid, which finally aggravates the infiltration of inflammation and severity of diseases [8, 33, 34]. Thus, high concentrations of soluble components of GLX in body fluid, especially CSF, implicates the damage degree of GLX, and indirectly the disruption of BBB. Here, our results showed dramatically increase of soluble HS and $\mathrm{HA}$ in plasma and CSF in the acute stage of NMO, MS and autoimmune GFAP astrocytopathy, indicating the degradation and disruption of GLX and BBB. Notably, the CSF levels of both HS and HA were positively associated with the severity of these three typical encephalomyelitis. Moreover, studies have announced that under inflammatory conditions, shedding of the GLX occurs in response to mediators and activation of MMPs [24, 25, 35, 36], resulting in release of fragments like HS and HA into the circulation. Our results also found the elevated expression of CSF MMP-1 in CSF and it positively associated with CSF HS or HA in NMO, MS and autoimmune GFAP astrocytopathy.

The current study also focused on the neuro-inflammation extent. Considerable evidence has implicated that the release of inflammation-mediated cytokines can stimulate the process of glycocalyx shedding [13, 15]. Of note, IL17A and IFN-y, two pro-inflammatory factors already proved to be involved in many autoimmune disorders, were activated NMO, MS and autoimmune GFAP astrocytopathy. Moreover, researchers have pointed out that MMPs can be induced under the inflammatory condition [37, 38]. Notably, a universal positive correlation was found between concentrations of CSF HS and that of IFN- $\gamma$, IL-17A, MMP-1 in these diseases. Therefore, the release of proinflammatory cytokines may interact with MMPs and result in glycocalyx dysfunction. Conversely, the disruption of endothelial GLX can cause damage to BBB and in turn exacerbate the extent of inflammation and the activation of MMPs.

Due to the difficulty of follow up and clinical situation in this study, some precise work has been limited, like dynamic monitoring the time duration of HS and HA shedding and assess the association between degree of glycocalyx destruction and disease severity in different course of these neurological disorders. Therefore, a further research in response to the above issues is worthy in the future clinical and animal experiments.

In conclusion, our findings demonstrated the glycocalyx degradation occurred in the acute stage of NMO, MS and autoimmune GFAP astrocytopathy, which was implicated by elevated shedding levels of HS and HA in plasma and CSF here. Moreover, CSF HS and HA were correlated with the severity of these diseases. Importantly, CSF HS and HA may act as potential indicators for the diagnosis of these three typical autoimmune encephalomyelitis. In addition, inflammation was also activated. Further investigations targeting measuring dynamic concentrations of glycocalyx shedding in different courses of diseases are worthy. Importantly, therapeutic strategy for protecting GLX may be effective to these diseases.

\section{Declarations}

Ethics approval and consent to participate 
This study was approved by the Ethics Committee of the Nan Fang Hospital and all the above subjects have signed the informed consent.

Consent for publication

Not applicable.

Availability of data and material

All data generated or analysed during this study are included in this published article.

Conflict of interest

No conflict of interest.

Funding

Financial support came from the National Natural Science Foundation of China $(81301028,81673950)$, Guangdong Provincial Science and Technology plan projects (2017A020215182), and Natural Science Foundation of Guangdong Provine (2019A1515011434). Xinjiang Uygur Autonomous Region Science and Technology Support Project (2018E02099).

Authors' contributions

Jinyu Chen and Honghao Wang conceived this study and designed the experiments. Shanshan Pei, Jiajia Zhu, Zheyi Zhou, and Zhanhang Wang collected the samples and clinical data. Shanshan Pei, Yuewen Ding, and Yu Peng performed the experiments, analyzed the data, and wrote the manuscript. Shanshan Pei re-performed all the data analyses and revised the manuscript. All authors read and approved the final version of the manuscript and agreed to submit it for publication.

Acknowledgements

Not applicable.

\section{References}

[1] Lucchinetti CF, Guo Y, Popescu BF, Fujihara K, Itoyama Y, Misu T. The pathology of an autoimmune astrocytopathy: lessons learned from neuromyelitis optica. Brain Pathol. 2014;24(1):83-97.

[2] Kawachi I, Lassmann H. Neurodegeneration in multiple sclerosis and neuromyelitis optica. J Neurol Neurosurg Psychiatry. 2017;88:137-45.

[3] Fang B, McKeon A, Hinson SR, Kryzer TJ, Pittock SJ, Aksamit AJ, et al. Autoimmune Glial Fibrillary Acidic Protein Astrocytopathy: A Novel Meningoencephalomyelitis. JAMA Neurol. 2016;73:1297-307.

[4] Diamond B, Honig G, Mader S, Brimberg L, Volpe BT. Brain-reactive antibodies and disease. Annu Rev Immunol. 2013;31:345-85. 
[5] Hausser-Kinzel S, Weber MS. The Role of B Cells and Antibodies in Multiple Sclerosis, Neuromyelitis Optica, and Related Disorders. Front Immunol. 2019;10:201.

[6] Banks WA. The blood-brain barrier in neuroimmunology: Tales of separation and assimilation. Brain Behav Immun. 2015;44:1-8.

[7] Varatharaj A, Galea I. The blood-brain barrier in systemic inflammation. Brain Behav Immun. 2017;60:1-12.

[8] Stewart PA. Endothelial vesicles in the blood-brain barrier: are they related to permeability? Cell Mol Neurobiol. 2000;20(2):149-63.

[9] Ortiz GG, Pacheco-Moises FP, Macias-Islas MA, Flores-Alvarado LJ, Mireles-Ramirez MA, Gonzalez-Renovato ED, et al. Role of the blood-brain barrier in multiple sclerosis. Arch Med Res. 2014;45:687-97.

[10] Reitsma S, Slaaf DW, Vink H, van Zandvoort MA, oude Egbrink MG. The endothelial glycocalyx: composition, functions, and visualization. Pflugers Arch. 2007;454:345-59.

[11] Weinbaum S, Tarbell JM, Damiano ER. The structure and function of the endothelial glycocalyx layer. Annu Rev Biomed Eng. 2007;9:121-67.

[12] Curry FE, Adamson RH. Endothelial glycocalyx: permeability barrier and mechanosensor. Ann Biomed Eng. 2012;40:828-39.

[13] Kolarova H, Ambruzova B, Svihalkova Sindlerova L, Klinke A, Kubala L. Modulation of endothelial glycocalyx structure under inflammatory conditions. Mediators Inflamm. 2014;2014:694312.

[14] Zeng Y. Endothelial glycocalyx as a critical signalling platform integrating the extracellular haemodynamic forces and chemical signalling. J Cell Mol Med. 2017;21:1457-62.

[15] Zhang X, Sun D, Song JW, Zullo J, Lipphardt M, Coneh-Gould L, et al. Endothelial cell dysfunction and glycocalyx - A vicious circle. Matrix Biol. 2018;71-72:421-31.

[16] Rabelink TJ, de Zeeuw D. The glycocalyx-linking albuminuria with renal and cardiovascular disease. Nat Rev Nephrol. 2015;11:667-76.

[17] Zhu J, Li X, Yin J, Hu Y, Gu Y, Pan S. Glycocalyx degradation leads to blood-brain barrier dysfunction and brain edema after asphyxia cardiac arrest in rats. J Cereb Blood Flow Metab. 2018;38:1979-92.

[18] Zhu J, Li Y, Zheng D, Wang Z, Pan S, Yin J, et al. Elevated Serum and Cerebrospinal Fluid CD138 in Patients With Anti-N-Methyl-d-Aspartate Receptor Encephalitis. Front Mol Neurosci. 2019;12:116.

[19] Pei S, Zheng D, Wang Z, Hu X, Pan S, Wang H. Elevated soluble syndecan-1 levels in neuromyelitis optica are associated with disease severity. Cytokine. 2018;111:140-5.

[20] DellaValle B, Manresa-Arraut A, Hasseldam H, Stensballe A, Rungby J, Larsen A, et al. Detection of Glycan Shedding in the Blood: New Class of Multiple Sclerosis Biomarkers? Front Immunol. 2018;9:1254.

[21] Tarbell JM, Cancel LM. The glycocalyx and its significance in human medicine. J Intern Med. 2016;280:97-113. 
[22] Mende M, Bednarek C, Wawryszyn M, Sauter P, Biskup MB, Schepers U, et al. Chemical Synthesis of Glycosaminoglycans. Chem Rev. 2016;116:8193-255.

[23] Kothur K, Wienholt L, Brilot F, Dale RC. CSF cytokines/chemokines as biomarkers in neuroinflammatory CNS disorders: A systematic review. Cytokine. 2016;77:227-37.

[24] Ali MM, Mahmoud AM, Le Master E, Levitan I, Phillips SA. Role of matrix metalloproteinases and histone deacetylase in oxidative stress-induced degradation of the endothelial glycocalyx. Am J Physiol Heart Circ Physiol. 2019;316:H647-H63.

[25] Lipowsky $\mathrm{HH}$. The endothelial glycocalyx as a barrier to leukocyte adhesion and its mediation by extracellular proteases. Ann Biomed Eng. 2012;40:840-8.

[26] Jiang X, Andjelkovic AV, Zhu L, Yang T, Bennett MVL, Chen J, et al. Blood-brain barrier dysfunction and recovery after ischemic stroke. Prog Neurobiol. 2018;163-164:144-71.

[27] Uchimido R, Schmidt EP, Shapiro NI. The glycocalyx: a novel diagnostic and therapeutic target in sepsis. Crit Care. 2019;23:16.

[28] Thompson AJ, Banwell BL, Barkhof F, Carroll WM, Coetzee T, Comi G, et al. Diagnosis of multiple sclerosis: 2017 revisions of the McDonald criteria. Lancet Neurol. 2018;17:162-73.

[29] Tan CT, Mao Z, Qiu W, Hu X, Wingerchuk DM, Weinshenker BG. International consensus diagnostic criteria for neuromyelitis optica spectrum disorders. Neurology. 2016;86:491-2.

[30] Lipowsky HH. Protease Activity and the Role of the Endothelial Glycocalyx in Inflammation. Drug Discov Today Dis Models. 2011;8:57-62.

[31] Platt MP, Agalliu D, Cutforth T. Hello from the Other Side: How Autoantibodies Circumvent the Blood-Brain Barrier in Autoimmune Encephalitis. Front Immunol. 2017;8:442.

[32] Kutuzov N, Flyvbjerg H, Lauritzen M. Contributions of the glycocalyx, endothelium, and extravascular compartment to the blood-brain barrier. Proc Natl Acad Sci U S A. 2018;115:E9429-E38.

[33] Chelazzi C, Villa G, Mancinelli P, De Gaudio AR, Adembri C. Glycocalyx and sepsis-induced alterations in vascular permeability. Crit Care. 2015;19:26.

[34] Rahbar E, Cardenas JC, Baimukanova G, Usadi B, Bruhn R, Pati S, et al. Endothelial glycocalyx shedding and vascular permeability in severely injured trauma patients. J Transl Med. 2015;13:117.

[35] Rempe RG, Hartz AMS, Bauer B. Matrix metalloproteinases in the brain and blood-brain barrier: Versatile breakers and makers. J Cereb Blood Flow Metab. 2016;36:1481-507.

[36] Rosenberg GA. Matrix metalloproteinases and neuroinflammation in multiple sclerosis. Neuroscientist. 2002;8:586-95.

[37] Fingleton B. Matrix metalloproteinases as regulators of inflammatory processes. Biochim Biophys Acta Mol Cell Res. 2017:2036-42. 


\section{Figures}
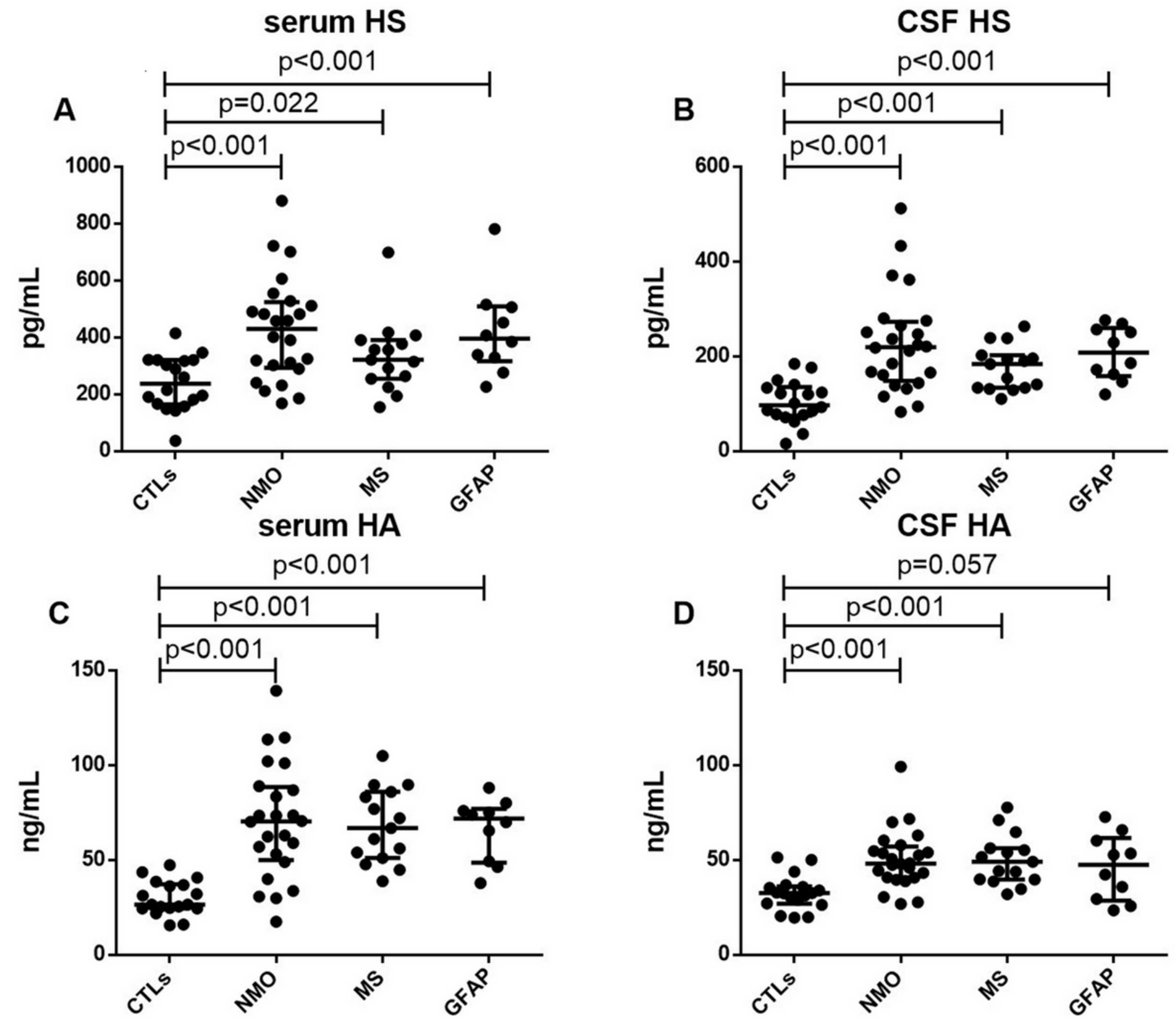

Figure 2

Serum and CSF levels of HS and HA. HS (A), HA (C) levels in the serum of controls (CTLs) and patients with NMO, MS and GFAP encephalitis; HS (B), HA (D) levels in the CFS of controls (CTLs) and patients with NMO, MS and GFAP encephalitis. The $\mathrm{p}$-values were indicated within each analysis. 
A

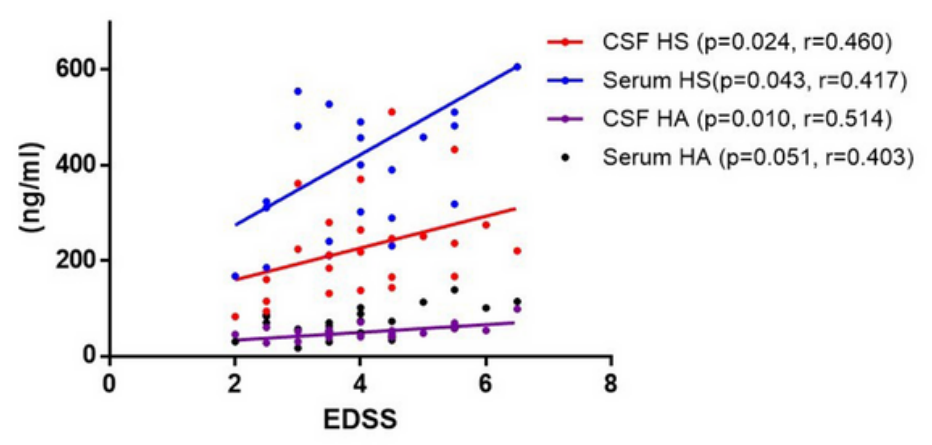

C

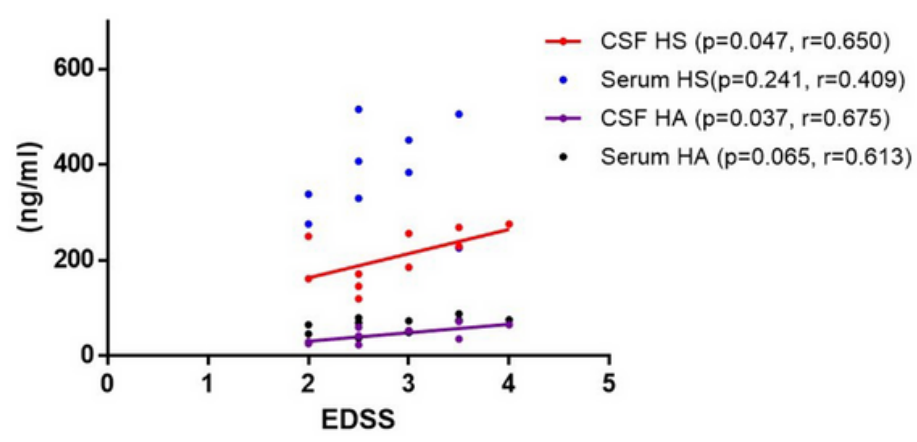

B MS

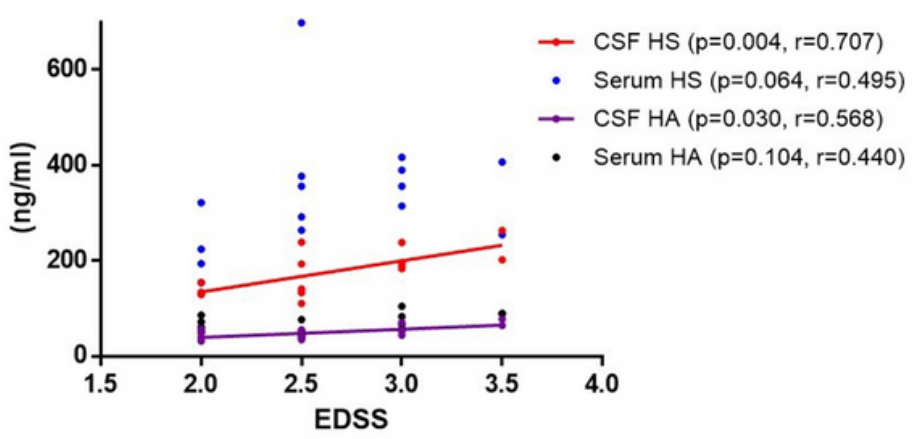

\section{Figure 3}

Correlation between the levels of both CSF and plasma HS, HA and EDSS scores of patients with NMO, MS and GFAP encephalitis. (A) In NMO, the levels of HS and HA in CSF and plasma HS were positively correlated with EDSS scores, while no correlation was found between plasma HA levels and EDSS scores. (B) In MS, the levels of HS and HA in CSF were significantly correlated with EDSS scores, while no correlation was found between plasma HS, HA levels and EDSS scores. (C) In autoimmune GFAP astrocytopathy, the levels of HS and HA in CSF were significantly correlated with EDSS scores, while no correlation was found between plasma HS and HA levels and EDSS scores. The $p$ and r-values were indicated within each analysis. 
A

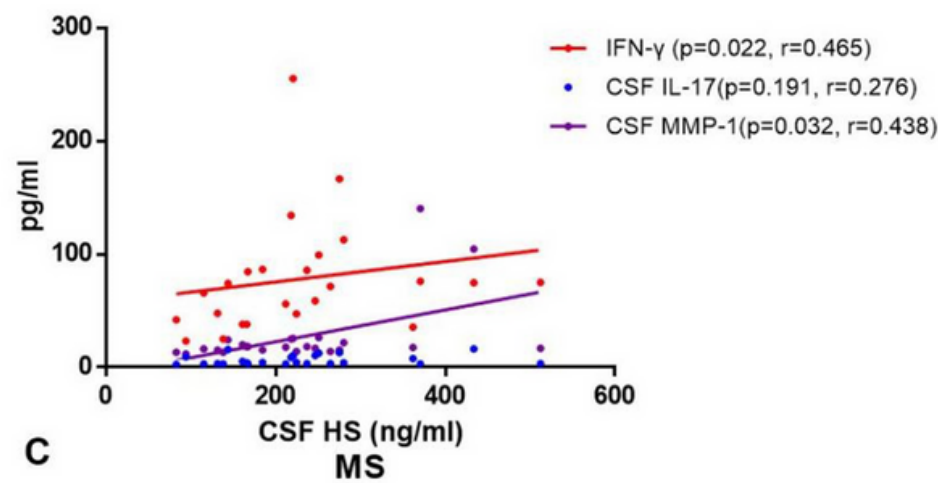

B NMO
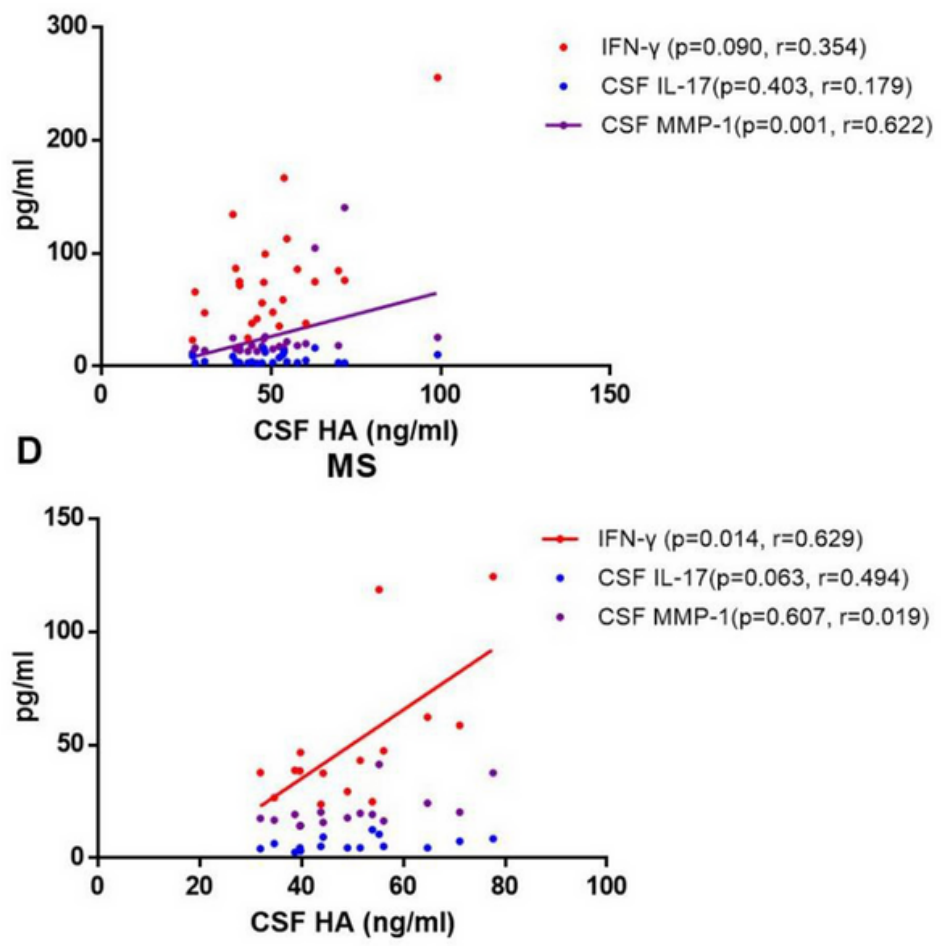

$\mathrm{F}$

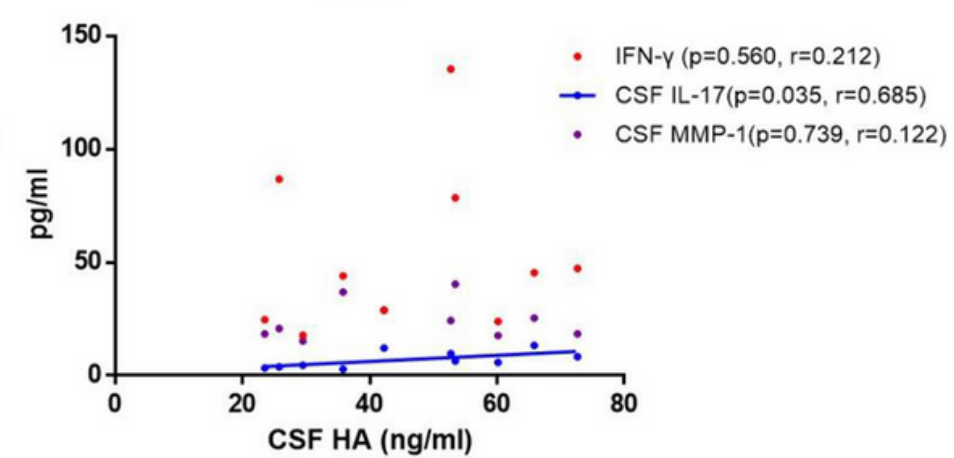

\section{Figure 5}

Correlation between the CSF levels of HA, HS and IFN- $y$, IL-17A, MMP-1 patients with NMO, MS and autoimmune GFAP astrocytopathy. The $p$ and r-values were indicated within each analysis. 

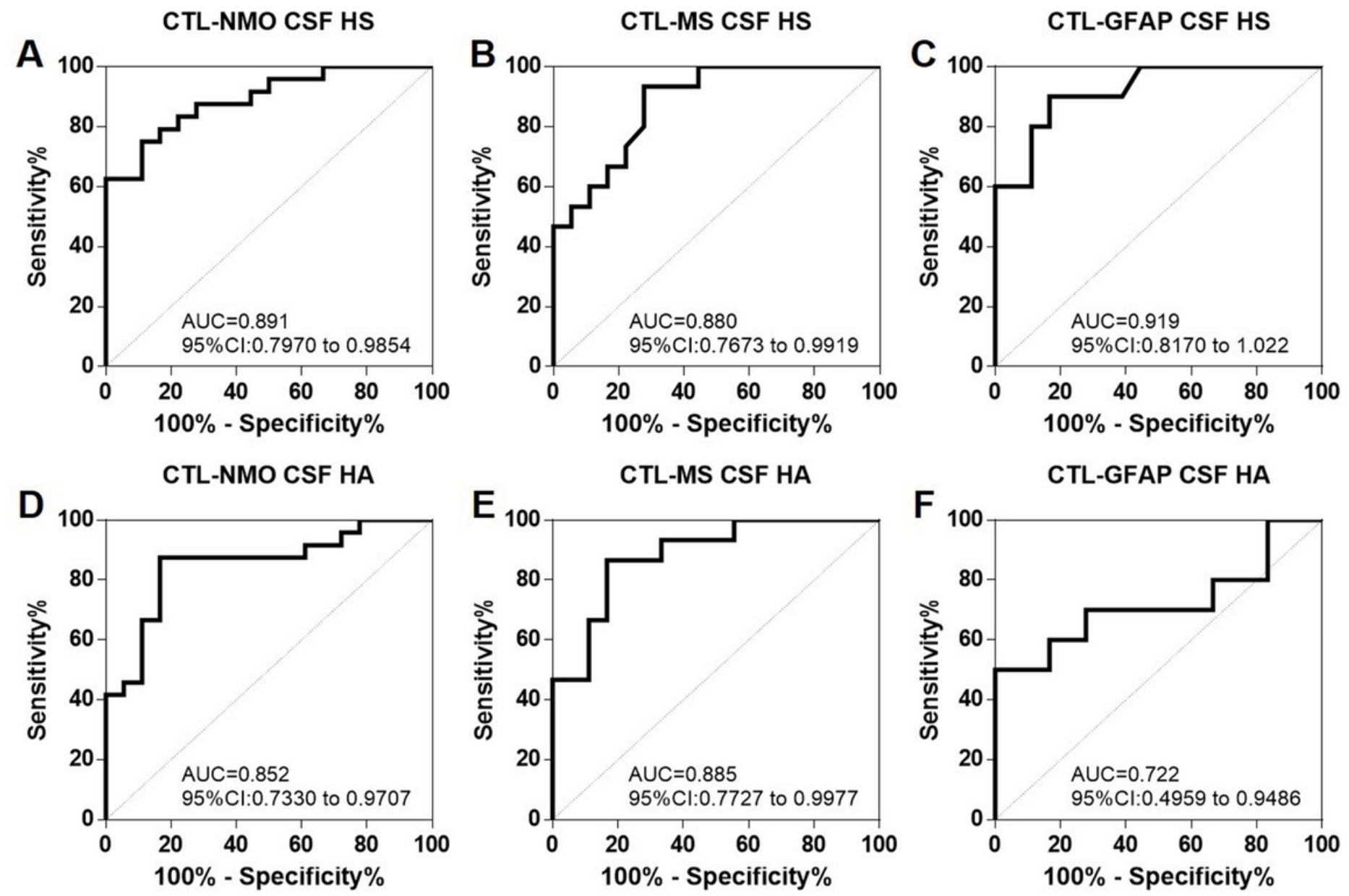

Figure 8

ROC curve analysis of CSF HS and HA in patients with NMO, MS and GFAP. (A, D) Diagnostic values of CSF HS and $\mathrm{HA}$ for distinguishing NMO and controls. (B, E) Diagnostic values of CSF HS and HA for distinguishing MS and controls. (C, F) Diagnostic values of CSF HS and HA for distinguishing GFAP and controls. AUC: area under the curve; $\mathrm{Cl}$, confidence interval. CTL: controls.

\section{Supplementary Files}

This is a list of supplementary files associated with this preprint. Click to download.

- Supplementarymaterial.pdf

- Supplementarymaterial.pdf 